Auroe: Atividade das enzimas

MárCia Marly Winck YAMAMOto

ORIENTADOR:

Prof. Dr. Sebastião Frettas de Mederos

\title{
corticoesteroidogênicas em pacientes normoandrogênicas e hiperandrogênicas com síndrome dos ovários policísticos
}

\section{Activities of the corticosteroidogenic enzimes in normoandrogenic and hiperandrogenic patients with polycystic ovary syndrome}

\section{Resumo de Tese}

Palavras-chave

Síndrome dos ovários policísticos esteroidogênese hiperadrogenismo

Keywords

Polycystic ovary syndrome Steroidogenesis Hyperandrogenism
Dissertação apresentada ao programa de Pós-graduação em Ciências da Saúde, Área de Concentração Reprodução Humana e Climatério, linha de pesquisa Endocrinologia e Climatério - Universidade Federal de Mato Grosso - Faculdade de Medicina, em 29 de junho de 2012.

OBJETIVO: Avaliar a atividade das enzimas corticoesteroidogênicas em mulheres com síndrome dos ovários policísticos (SOP). MÉTODOS: Estudo de coorte, incluindo 122 pacientes com SOP; 81 apresentavam hiperandrogenismo bioquímico e 41 tinham níveis de androgênios normais. Atividade das enzimas corticoesteroidogênicas, examinadas pela razão produto/precursor, foram comparadas entre pacientes normoandrogênicas e hiperandrogênicas, tanto em condições basais como após estimulação adrenal com cortrosina. RESULTADOS: Duas pacientes, uma de cada grupo, foram excluídas por hiperplasia congênita de supra renal não clássica. Não se observou diferença na idade ou variáveis antropométricas entre pacientes com normo ou hiperandrogênicas. Pacientes hiperandrogênicas tiveram níveis elevados de colesterol total e insulina, maior produção de insulina pelas células $\beta$ pancreáticas e níveis baixos de colesterol ligado a lipoproteína de alta densidade, quando comparadas com as normoandrogênicas. Atividades da 17a-hidroxilase e 17,20 liase foram equivalentes na via $\Delta 5$ em ambos os grupos de pacientes com SOP. Na via $\Delta 4$, em condições basais, pacientes hiperandrogênicas tiveram maior atividade da 17a-hidroxilase e 17,20 liase $(p=0,005$ e $p=0,04$, respectivamente). Pacientes hiperandrogênicas apresentaram diminuição da atividade da 21 a-hidroxilase e $11 \beta$-hidroxilase $(p \leq 0,001)$ e aumento da $3 \beta$-hidroxiesteroide desidrogenase $(3 \beta$-HSD) na conversão de DHEA em androstenediona (A). Atividades da aromatase e 17ß-hidroxiesteroide desidrogenase (17 $\beta$-HSD) foram diminuídas no grupo de SOP com hiperandrogenismo. Após estimulação com cortrosina, atividade da 17,20 liase persistiu aumentada na via $\Delta 4$ ( $p<0,001$ ). CONCLUSÕES: Na via $\Delta 5$, as atividades da $17 a$-hidroxilase e 17,20 liase foram equivalentes em ambos os grupos de pacientes mas, na via $\Delta 4$, a atividade da 17,20 liase foi maior no grupo com hiperadrogenismo. $3 \beta$-HSD foi mais ativa na conversão de DHEA para A, enquanto 21 a-hidroxilase e $11 \beta$-hidroxilase, foram menos ativas em pacientes hiperandrogênicas. Após estimulação com cortrosina, somente atividade da 17,20 liase persistiu elevada na via $\Delta 4$ 